\title{
Analysis of Demographics and Outcomes of Surgical Resection in the Central Nervous System of Patients With Metastatic Melanoma
}

\author{
Achuta Kumar Guddatia, c, Hector Picon ${ }^{\mathrm{b}}$
}

\begin{abstract}
Background: Patients with melanoma frequently develop central nervous system metastases. Oligometastatic disease is often treated either by surgical resection or by stereotactic radiotherapy. This study investigates the trends and clinical outcomes of patients with melanoma who have undergone surgical procedures on the central nervous system during their hospitalization.
\end{abstract}

Methods: A retrospective study was performed based on admissions of adult patients who underwent craniotomy for metastatic melanoma from 2000 to 2014 using the Nationwide Inpatient Sample database. The primary outcome measure was all-cause in-hospital mortality. Secondary outcomes included hospital length of stay (LOS) and discharge disposition (home/home with health care and skilled nursing facilities/ long-term acute care (SNFs/LTAC)). Factors associated with in-hospital mortality were examined by multivariable logistic regression. We adjusted for patient and hospital characteristics, payer, and comorbid conditions. We also examined trends of mortality for the study years.

Results: There were an estimated 1,216 discharges of patients with melanoma undergoing craniotomy during the study period. Patients undergoing surgical interventions were typically males (69\%) and whites $(79 \%)$. Ninety-eight percent of procedures were performed at teaching hospitals. Unadjusted all-cause in-hospital mortality was 3.1\%. There was no significant difference in mortality over 13 years. Age, gender, and race were not associated with increased in-hospital mortality. LOS was longer in elderly and those with higher Charlson Comorbidity Index. Of the survivors, $76 \%$ were discharged to home or with home healthcare while $24 \%$ were discharged to SNFs/LTAC. Patients with age $>65$ (odds ratio (OR): $2.9 ; 95 \%$ confidence interval (CI): 2.2 - 3.9, $\mathrm{P}<0.001$ ) and those with higher Charlson Comorbidity Index (OR: 1.2; 95\% CI: 1.1 - 1.3) had higher odds for being discharged to SNFs/LTAC.

Manuscript submitted August 16, 2021, accepted August 30, 2021

Published online December 8, 2021

aDivision of Hematology/Oncology, Georgia Cancer Center, Augusta University, Augusta, GA 30912, USA

bMedical College of Georgia, Augusta University, Augusta, GA 30912, USA ${ }^{\mathrm{c} C}$ Corresponding Author: Achuta Kumar Guddati, Division of Hematology/ Oncology, Georgia Cancer Center, Augusta University, Augusta, GA 30909, USA. Email: aguddati@augusta.edu

doi: https://doi.org/10.14740/wjon1408
Conclusions: Patients who undergo craniotomy for metastatic melanoma have a low in-hospital mortality rate. One quarter of patients were discharged to SNFs/LTAC.

Keywords: Craniotomy; Melanoma; Morbidity; Mortality

\section{Introduction}

The 5-year survival for melanoma is promising, estimated at $92 \%$, and is even as high as $98 \%$ for those diagnosed at an early stage [1]. However, the presence of brain metastasis is known to be a very poor prognostic indicator, dropping the 5 -year survival to as low as $2.3 \%$ [2]. It is known to lead to death in a majority of patients recognized to have this pattern of metastasis $[3,4]$. Brain metastases have been found in as much as $80 \%$ autopsies of patients with metastatic melanoma, underscoring the importance and frequency of this complication [5]. Median overall survival after diagnosis has been measured at 4.7 months [6]. Brain metastasis in melanoma has been labeled as radioresistant given that it shows subpar response rates to conventionally fractionated radiation therapy $[7,8]$. Further, these patients are often excluded from clinical trials due to the poor prognosis of their disease [9]. Other studies suggest that stereotactic radiosurgery (STR) for brain metastasis could be more beneficial if the patients are selected carefully based on predictors of response, but secondary melanoma tumors may bleed significantly causing local failure of the treatment [10, 11]. Due to the imperfect and complicated nature of STR, other avenues of treatment need to be explored and studied.

When considering metastasis, it is important to consider the extent to which the cancer has colonized distant sites. A recent consensus study of European experts solidified the definition of oligometastatic disease as a diagnosis of limited metastatic disease based on imaging findings, usually accepted as up to four metastatic lesions $[12,13]$. These patients are considered to be candidates for craniotomy, the removal of the tumor via surgical opening of the skull; but the decision to undergo surgery is influenced by many other factors including age, lactate dehydrogenase levels, location of metastases, and symptoms $[13,14]$. Notably, it has also been shown that craniotomy combined with STR can greatly improve prognosis of melanoma patients with brain metastasis [14]. 
Craniotomy, however, is inherently risky due to the invasive nature of the procedure. A major complication is infection, with one study reporting that surgical infection risk was increased in patients who are male or who are undergoing elective (nontraumatic) craniotomy, both of which are relevant to this study [15]. Perioperative seizures are another risk, and are more common in patients diagnosed with a brain tumor [16]. Because craniotomy is an effective but invasive treatment option for patients with melanoma, the available data must be analyzed to be able to predict which patients will respond well to this therapy and which are at highest risk of mortality. Here, we present a retrospective cohort study on the trends and outcomes of patients with melanoma who undergo procedures on the central nervous system (CNS).

\section{Materials and Methods}

\section{Data source and study population}

The National (Nationwide) Inpatient Sample (NIS) is a large online database developed as part of the Healthcare Cost and Utilization Project for the purpose providing data to inform decision making across the various subfields of healthcare in the USA. It contains data on more than 7 million hospital stays stretching as far back as 1988 and represents the largest collection of longitudinal hospital data in the country. Approximately $20 \%$ of all inpatient admissions to acute care hospitals in the USA are represented in this data set. The admissions are weighted in order to make the data generalizable to all hospitals in the USA. Reported in the NIS is information about patient outcomes, diagnoses, quality, and charges. Hospital data include region, urban vs. rural, teaching status, and hospital size. Beginning in October of 2015, the medical coding system used by the NIS switched to International Classification of Disease, 10th Revision, Procedure Classification System (ICD-10-PCS) codes. Prior to that, the data came from International Classification of Diseases, Ninth Revision, Clinical Modification (ICD-9-CM) codes, which have a separate organizational structure. These changes were accounted for in the analysis presented in this study, which analyzed craniotomies in for metastatic melanoma from 2002 to 2014 .

\section{Outcomes}

The primary outcome measured was all-cause in-hospital mortality. The secondary outcomes were length of hospital stay (LOS) and discharge disposition. The options for discharge disposition were home, home with health care, and skilled nursing facilities/long-term acute care (SNFs/LTAC).

\section{Statistical analysis}

The data were analyzed using descriptive statistics. Differences in baseline patient characteristics such as race, gender, and age were identified, as were baseline hospital characteristics such as location, size, and type. These differences were analyzed separately to see if there was any correlation with differences in measured outcomes and minimize sampling bias. Race was self-reported. Primary payers included private, Medicare, Medicaid, self-pay, and others. Comparisons were made between groups using Pearson $\chi^{2}$ tests. Factors associated with in-hospital mortality were examined by multivariable logistic regression. The Charlson Comorbidity Index (CCI), which measures a patient's health status based on chronic conditions, was used to assess comorbidities $[17,18]$. Odds ratios and $95 \%$ confidence interval (CI) were estimated by survey logistic regression analyses for associations between variables. Independent predictors of mortality were determined using these analyses. P was kept at 0.05 . All statistical analysis was performed uesing STATA software version 14.2 (College Station, TX).

The study was approved by the Institutional Review Board (IRB); and was conducted in compliance with the ethical standards of the responsible institution on human subjects, as well as with the Helsinki Declaration.

\section{Results}

\section{Demographics of patients and hospitals}

From the years 2000 to 2014, approximately 1,216 patients were discharged following craniotomy for melanoma. The majority of the patients were males $(69.4 \%)$ versus females $(30.6 \%)$. The most common race was white $(79.4 \%)$ versus nonwhite $(20.6 \%)$. Most patients were under the age of 65 $(64.4 \%)$ versus over $65(35.6 \%)$. Most procedures were performed at large hospitals $(75.0 \%)$ whereas medium and smaller hospitals represented a much smaller proportion of cases (15.1\% and 9.3\%, respectively). The most common census divisions were Pacific (5.4\%) and South Atlantic (5.1\%), though the vast majority was unknown $(72.4 \%)$. The least represented census divisions were New England (1.6\%), Southeast (1.7\%) and Mountain (1.7\%). Most hospitals were teaching hospitals (70.6\%), with urban hospitals representing $19 \%$ and rural nonteaching representing only $1.8 \%$. The majority were located in the Southern USA (38.3\%), followed by West $(26.0 \%)$, Midwest $(18.5 \%)$, and Northeast $(17.2 \%)$. The primary expected payer was private insurance $(49.0 \%)$, while Medicare was second at $34.6 \%$ and Medicaid paid for only $9.5 \%$ of the reviewed procedures. Most patients were discharged to home $(59.5 \%)$, with SNFs/LTCF being the second most common discharge disposition $(22.9 \%)$ and home health as third most common (14.1\%). Most patients had a CCI of $>6(90.1 \%)$, while $2.2 \%$ had a CCI of $4-6$ and $7.6 \%$ had a CCI of $1-3$. The data were summarized in Table 1.

\section{Mortality}

Multivariable logistic regression was used to determine if demographic, clinical, or hospital features had an effect on the 
Table 1. Characteristics of Patients With Metastatic Melanoma Who Underwent Craniotomy

\begin{tabular}{|c|c|}
\hline Characteristic & Patients, n (\%) \\
\hline \multicolumn{2}{|l|}{ Sex } \\
\hline Male & $844(69.4 \%)$ \\
\hline Female & $372(30.6 \%)$ \\
\hline \multicolumn{2}{|l|}{ Age } \\
\hline$<65$ & $783(64.4 \%)$ \\
\hline$>65$ & $433(35.6 \%)$ \\
\hline \multicolumn{2}{|l|}{ Race } \\
\hline White & $965(79.4 \%)$ \\
\hline Nonwhite & $251(20.6 \%)$ \\
\hline \multicolumn{2}{|l|}{ Hospital bed size } \\
\hline Small & $113(9.3 \%)$ \\
\hline Medium & $184(15.1 \%)$ \\
\hline Large & $912(75.0 \%)$ \\
\hline Unknown & $7(0.6 \%)$ \\
\hline \multicolumn{2}{|l|}{ Census division } \\
\hline New England & $19(1.6 \%)$ \\
\hline Middle Atlantic & $37(3.0 \%)$ \\
\hline Northeast & $53(4.4 \%)$ \\
\hline Northwest & $23(1.9 \%)$ \\
\hline South Atlantic & $62(5.1 \%)$ \\
\hline Southeast & $21(1.7 \%)$ \\
\hline Southwest & $34(2.8 \%)$ \\
\hline Mountain & $21(1.7 \%)$ \\
\hline Pacific & $66(5.4 \%)$ \\
\hline Unknown & $880(72.4 \%)$ \\
\hline \multicolumn{2}{|l|}{ Location/teaching status } \\
\hline Rural non-teaching & $22(1.8 \%)$ \\
\hline Urban & $231(19.0 \%)$ \\
\hline Teaching & $859(70.6 \%)$ \\
\hline Unknown & $104(8.6 \%)$ \\
\hline \multicolumn{2}{|l|}{ Region of hospital } \\
\hline Northeast & $209(17.2 \%)$ \\
\hline Midwest & $225(18.5 \%)$ \\
\hline South & $466(38.3 \%)$ \\
\hline West & $316(26.0 \%)$ \\
\hline \multicolumn{2}{|l|}{ Primary expected payer } \\
\hline Medicare & $421(34.6 \%)$ \\
\hline Medicaid & $115(9.5 \%)$ \\
\hline Private insurance & $596(49.0 \%)$ \\
\hline Self-pay & $45(3.7 \%)$ \\
\hline No charge & $2(0.2 \%)$ \\
\hline Other & $34(2.8 \%)$ \\
\hline
\end{tabular}

Table 1. Characteristics of Patients With Metastatic Melanoma Who Underwent Craniotomy - (continued)

\begin{tabular}{|c|c|}
\hline Characteristic & Patients, n (\%) \\
\hline Unknown & $3(0.2 \%)$ \\
\hline \multicolumn{2}{|c|}{ Discharge disposition } \\
\hline Home & $724(59.5 \%)$ \\
\hline Home health & $172(14.1 \%)$ \\
\hline SNFs/LTAC & $278(22.9 \%)$ \\
\hline Others & $4(0.3 \%)$ \\
\hline Unknown & $38(3.1 \%)$ \\
\hline \multicolumn{2}{|l|}{$\mathrm{CCI}$} \\
\hline $1-3$ & $93(7.6 \%)$ \\
\hline $4-6$ & $27(2.2 \%)$ \\
\hline$>6$ & $1,096(90.1 \%)$ \\
\hline \multicolumn{2}{|l|}{ Year } \\
\hline 2000 & $54(4.4 \%)$ \\
\hline 2001 & $44(3.6 \%)$ \\
\hline 2002 & $47(3.9 \%)$ \\
\hline 2003 & $60(4.9 \%)$ \\
\hline 2004 & $60(4.9 \%)$ \\
\hline 2005 & $72(5.9 \%)$ \\
\hline 2006 & $59(4.9 \%)$ \\
\hline 2007 & $91(7.5 \%)$ \\
\hline 2008 & $94(7.7 \%)$ \\
\hline 2009 & $82(6.7 \%)$ \\
\hline 2010 & $117(9.6 \%)$ \\
\hline 2011 & $100(8.2 \%)$ \\
\hline 2012 & $105(8.6 \%)$ \\
\hline 2013 & $99(8.1 \%)$ \\
\hline 2014 & $132(10.9 \%)$ \\
\hline
\end{tabular}

SNFs/LTAC: skilled nursing facilities/long-term acute care; CCl: Charlson Comorbidity Index.

patient's odds of mortality. All cause in-hospital mortality was $3.1 \%$. Age, sex, and race were not associated with increased mortality. The multivariable logistic regression that shows the effect of these patient characteristics on mortality is shown in Table 2. P values for age, sex, and race were $0.74,0.39$, and 0.95 , respectively. As per the cut-off of $\mathrm{P}<0.05$ for significance, none of these analyzed characteristics had a correlation with mortality.

There was no significant change in mortality over the 15 year study period from 2000 to 2014 . This is visually represented in Figure 1, where mortality is expressed as a percentage of total cases performed in the study period. The highest mortality in that period was $10.9 \%$ in 2014 and the lowest was $3.6 \%$ in 2001 . The changes in mortality over time were not statistically significant for $\mathrm{P}<0.05$. The hospital characteristics hospital size, census division, location/teaching status, 
Table 2. Multivariable Logistic Regression for the Influence of Various Patient and Hospital Characteristics on Mortality

\begin{tabular}{lllllll} 
Measure & Characteristic & Odds ratio & Standard error & $\mathbf{z}$ & $\mathbf{P}>|\mathbf{z}|$ & $\mathbf{9 5 \%}$ CI \\
\hline Mortality & Age $>$ 65 & 1.12 & 0.39 & 0.33 & 0.74 & $0.57-2.20$ \\
& Sex & 0.71 & 0.28 & -0.87 & 0.39 & $0.33-1.54$ \\
& White & 1.03 & 0.45 & 0.06 & 0.95 & $0.43-2.44$ \\
& Midwest & 0.60 & 0.34 & -0.91 & 0.37 & $0.20-1.80$ \\
& South & 0.48 & 0.23 & -1.55 & 0.12 & $0.19-1.21$ \\
& West & 0.93 & 0.42 & -0.16 & 0.87 & $0.39-2.24$ \\
& CCI & 1.00 & 0.08 & 0.03 & 0.97 & $0.86-1.17$ \\
& Cons & 0.05 & 0.04 & -3.52 & 0.00 & $0.01-0.26$ \\
\hline
\end{tabular}

Age, sex, and white are patient characteristics. Midwest, South, and West are hospital geographic locations. Cons was used to estimate baseline odds. P was considered significant if < 0.05. CCl: Charlson Comorbidity Index; Cl: confidence interval.

hospital region, primary expected payer, CCI, and discharge disposition were not associated with increased mortality (data not shown).

\section{Length of stay (LOS)}

LOS was examined for its characteristics and potential correlation with other variables. A longer LOS was significantly more likely in those age $>65$ and patients with a higher CCI. This analysis is presented in Table 3 . The other analyzed factors, which consisted of sex, race, region, and year, had no correlation with LOS for $\mathrm{P}>0.05$.

\section{Patient disposition}

The same analysis was conducted using discharge disposition. It was determined that discharge to SNFs/LTAC was associated with higher odds ratios for patients with age $>65$ (OR:
2.9; 95\% CI: $2.2-3.9, \mathrm{P}<0.001)$ and higher CCI (OR: 1.2; 95\% CI: 1.1 - 1.3). The data are shown in Table 4. There was no correlation between discharge to SNFs and patient/hospital characteristics of sex, race, region, or year.

\section{Discussion}

The prognosis of melanoma is highly dependent upon the stage at diagnosis. For the cases in which melanoma was not diagnosed early, metastasis to the CNS is a serious complication. The objective of this study was to analyze the characteristics and outcomes of patients who underwent craniotomy as treatment for metastatic melanoma. Here, we investigated over 1,200 cases of craniotomy in melanoma to attempt to identify relevant trends or predictors of mortality. This topic is of importance because there is conflicting literature regarding the utility of other means of predicting melanoma patient prognosis using tumor characteristics, such as tumor depth, location, and ulceration $[19,20]$. Our results indicate that

\section{Change in Mortality}

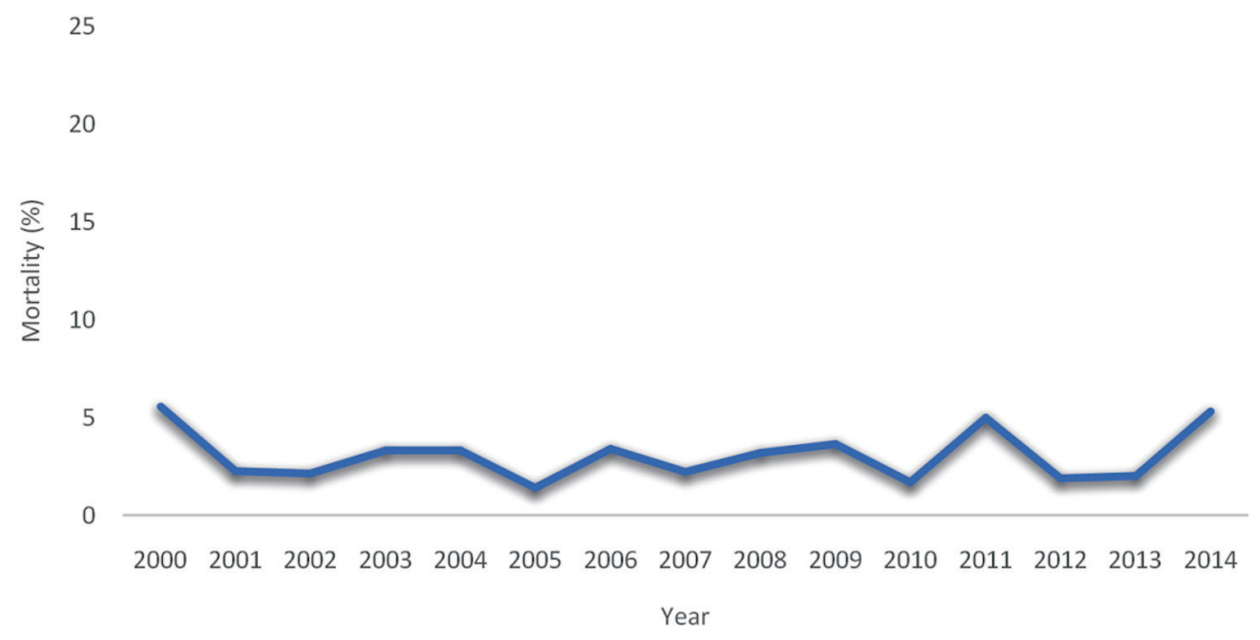

Figure 1. The change in mortality from the year 2000 to 2014 is shown. Mortality is expressed as a percentage of the total number of cases performed in the study period. 
Table 3. Multivariable Logistic Regression for the Influence of Various Patient and Hospital Characteristics on LOS

\begin{tabular}{lllllll}
\hline Measure & Characteristic & Odds ratio & Standard error & $\mathbf{z}$ & P $>|\mathbf{z}|$ & 95\% CI \\
\hline Ln(LOS) & Age $>65$ & 0.12 & 0.05 & 2.39 & 0.02 & $0.02-0.21$ \\
& Sex & 0.03 & 0.05 & 0.60 & 0.55 & $-0.07-0.13$ \\
& White & -0.01 & 0.06 & -0.21 & 0.83 & $-0.13-0.10$ \\
& Midwest & -0.09 & 0.08 & -1.18 & 0.24 & $-0.25-0.06$ \\
& South & -0.21 & 0.07 & -3.08 & 0.00 & $-0.34-(-0.07)$ \\
& West & -0.23 & 0.07 & -3.27 & 0.00 & $-0.37-(-0.09)$ \\
& CCI & 0.07 & 0.01 & 6.44 & 0.00 & $0.05-0.09$ \\
& Year & 0.00 & 0.01 & -0.56 & 0.58 & $-0.01-0.01$ \\
& Cons & 7.45 & 11.24 & 0.66 & 0.51 & $-14.60-29.4$ \\
\hline
\end{tabular}

Age, sex, and white are patient characteristics. Midwest, South, and West are hospital geographic locations. Cons was used to estimate baseline odds. P was considered significant if < 0.05. LOS: length of stay; $\mathrm{CCl}$ : Charlson Comorbidity Index; Cl: confidence interval.

there is no change in in-hospital mortality over the 13-year period analyzed in this study, and age, race, and gender had no impact on mortality. This is contrary to other studies, which have found that these characteristics are predictive for mortality in melanoma patients [21-23], though these studies were not specific for craniotomy or even metastatic melanoma. It is interesting that gender played no significant role in outcomes, given that the anatomic manifestations of melanoma are different in men and women [22]. A possible explanation is that metastatic melanoma behaves similarly irrespective of the gender of the patient, even if there are differences in the initial manifestation.

We also ran statistical analyses in search of other important correlations in this dataset. Patients of advanced age were more likely to have a longer LOS and were also more likely to be discharged to SNFs/LTAC. CCI was not found to predict mortality in the patients of this study, which is unexpected given that it is a strong predictor of cancer mortality and in-hospital mortality $[17,24]$. One explanation is that candidates for craniotomy must be chosen carefully, and while they do have metastatic melanoma, cannot be selected if they have widespread metastasis. Thus, while long-term survival for these patients is uncertain, their short-term survival is likely because of their lack of disseminated metastasis. This is further evidenced by the fact that most patients $(76 \%)$ were discharged to home rather than a locale that implies a less stable disposition such as SNF/LTAC.

Metastasectomy is a promising treatment option for patients with metastatic melanoma, but a minority of patients with stage IV melanoma qualify for this procedure [25]. Importantly, Li et al found that the most important prognostic indicator for melanoma was the number of distant organs with metastasis [21]. Therefore, it follows that the best candidates for metastasectomy are patients with oligometastasis confined to one organ. For those with disseminated disease, immunotherapeutic agents are being explored as treatment options. Nivolumab, a programmed death 1 (PD-1) checkpoint inhibitor, and ipilimumab, a cytotoxic T-lymphocyte-associated antigen 4 (CTLA-4) checkpoint inhibitor are both used, with nivolumab alone or nivolumab plus ipilimumab showing benefits for progression-free survival in previously untreated patients [26]. In patients with metastatic melanoma with a $B R A F$ mutation, a regimen of the drugs dabrafenib and trametinib have been shown to improve survival by targeting specific players in the mitogen-activated protein kinase (MAPK) pathway of cellular proliferation [27]. Though previously used as

Table 4. Multivariable Logistic Regression for the Influence of Various Patient and Hospital Characteristics on Disposition to SNFs

\begin{tabular}{|c|c|c|c|c|c|c|}
\hline Measure & Characteristic & Odds ratio & Standard error & $\mathbf{z}$ & $\mathbf{P}>|\mathbf{z}|$ & $95 \% \mathrm{CI}$ \\
\hline \multirow[t]{6}{*}{ SNFs } & Age $>65$ & 2.94 & 0.44 & 7.30 & 0.00 & $2.20-3.93$ \\
\hline & Sex & 1.26 & 0.20 & 1.46 & 0.14 & $0.92-1.72$ \\
\hline & Midwest & 1.39 & 0.33 & 1.42 & 0.16 & $0.88-2.20$ \\
\hline & South & 0.76 & 0.16 & -1.31 & 0.19 & $0.51-1.14$ \\
\hline & Year & 1.03 & 0.02 & 1.81 & 0.07 & $1.00-1.07$ \\
\hline & Cons & 0.00 & 0.00 & -1.90 & 0.06 & $0.00-10.21$ \\
\hline
\end{tabular}

Age, sex, and white are patient characteristics. Midwest, South, and West are hospital geographic locations. Cons was used to estimate baseline odds. P was considered significant if < 0.05. SNFs: skilled nursing facilities; CCl: Charlson Comorbidity Index; Cl: confidence interval. 
treatment, cytotoxic chemotherapy and whole brain irradiation no longer have a role in metastatic melanoma treatment [28]. Importantly, Wollina and Brzezinski recently re-evaluated the role of metastasectomy in the new era of immunotherapy and found that metastasectomy remains effective for qualifying patients, though they suggest that modern systemic therapy could be used in conjunction with craniotomy to improve survival [29].

This study had many important strengths and limitations. The biggest advantage to using NIS data is the sample size, as we were able to analyze a diverse patient population with representation from hospitals across the nation. Significant limitations include the retrospective nature of the study, because the specific reason for a correlation cannot always be identified in this kind of study, as well as the potential for ICD-9 miscoding or underreporting by clinicians or hospitals. Further, there is no ability to follow up with patients after their discharge from the hospital, and some patients could have been counted twice since the data are not based on individuals but instead on admissions and procedures.

\section{Conclusions}

Using the large national database known as the NIS, this study analyzes the trends and clinical outcomes of patients undergoing craniotomy for metastatic melanoma. To the best of our knowledge, this is the first study to analyze the outcomes for this patient population during this time period. It was found that age, gender, and race had no influence on in-hospital mortality. In fact, the in-hospital mortality rate was found to be overall low for these patients at 3.1\%. The LOS was longer in elderly patients and patients with a higher CCI. Finally, most patients were discharged to home, but those with advanced age or higher CCI were more likely to be discharged to SNFs/ LTAC. Future studies should collect more data on patient demographics (e.g., socioeconomic status, history of alcohol/tobacco use) and disease characteristics (e.g., stage at diagnosis, tumor markers) to attempt to further stratify patients into subgroups which can then be analyzed for clinically relevant predictors of in-hospital morality in these patients. Additionally, long-term follow-up is needed with these patients to identify predictors of survival.

\section{Acknowledgments}

This work was presented as an abstract at ASCO 2021.

\section{Financial Disclosure}

The authors declare that there was no funding for this study.

\section{Conflict of Interest}

None to declare.

\section{Informed Consent}

Informed consents were obtained.

\section{Author Contributions}

Hector Picon and Achuta Kumar Guddati: study design, data analysis, and manuscript writing. The authors have read the manuscript and agree to the content.

\section{Data Availability}

The data supporting the findings of this study are available from the corresponding author upon reasonable request.

\section{References}

1. Miller KD, Siegel RL, Lin CC, Mariotto AB, Kramer JL, Rowland JH, Stein KD, et al. Cancer treatment and survivorship statistics, 2016. CA Cancer J Clin. 2016;66(4):271-289.

2. Hall WA, Djalilian HR, Nussbaum ES, Cho KH. Longterm survival with metastatic cancer to the brain. Med Oncol. 2000;17(4):279-286.

3. Weide B, Richter S, Buttner P, Leiter U, Forschner A, Bauer J, Held L, et al. Serum S100B, lactate dehydrogenase and brain metastasis are prognostic factors in patients with distant melanoma metastasis and systemic therapy. PLoS One. 2013;8(11):e81624.

4. Sampson JH, Carter JH, Jr., Friedman AH, Seigler HF. Demographics, prognosis, and therapy in 702 patients with brain metastases from malignant melanoma. J Neurosurg. 1998;88(1):11-20.

5. Kenchappa RS, Tran N, Rao NG, Smalley KS, Gibney GT, Sondak VK, Forsyth PA. Novel treatments for melanoma brain metastases. Cancer Control. 2013;20(4):298306.

6. Davies MA, Liu P, McIntyre S, Kim KB, Papadopoulos N, Hwu WJ, Hwu P, et al. Prognostic factors for survival in melanoma patients with brain metastases. Cancer. 2011;117(8):1687-1696.

7. Chang EL, Selek U, Hassenbusch SJ, 3rd, Maor MH, Allen PK, Mahajan A, Sawaya R, et al. Outcome variation among "radioresistant" brain metastases treated with stereotactic radiosurgery. Neurosurgery. 2005;56(5):936945; discussion 936-945.

8. Brown PD, Brown CA, Pollock BE, Gorman DA, Foote RL. Stereotactic radiosurgery for patients with "radioresistant" brain metastases. Neurosurgery. 2002;51(3):656665; discussion 665-657.

9. Cohen JV, Tawbi H, Margolin KA, Amravadi R, Bosenberg M, Brastianos PK, Chiang VL, et al. Melanoma central nervous system metastases: current approaches, challenges, and opportunities. Pigment Cell Melanoma Res. 2016;29(6):627-642. 
10. Kim H, Jung TY, Kim IY, Jung S, Moon KS, Park SJ. The usefulness of stereotactic radiosurgery for radioresistant brain metastases. J Korean Neurosurg Soc. 2013;54(2):107-111.

11. Sayan M, Zoto Mustafayev T, Sahin B, Kefelioglu ESS, Wang SJ, Kurup V, BalmukA, et al. Evaluation of response to stereotactic radiosurgery in patients with radioresistant brain metastases. Radiat Oncol J. 2019;37(4):265-270.

12. Guckenberger M, Lievens Y, Bouma AB, Collette L, Dekker A, deSouza NM, Dingemans AC, et al. Characterisation and classification of oligometastatic disease: a European Society for Radiotherapy and Oncology and European Organisation for Research and Treatment of Cancer consensus recommendation. Lancet Oncol. 2020;21(1):e18-e28.

13. Gutzmer R, Vordermark D, Hassel JC, Krex D, Wendl C, Schadendorf D, Sickmann T, et al. Melanoma brain metastases - Interdisciplinary management recommendations 2020. Cancer Treat Rev. 2020;89:102083.

14. Eigentler TK, Figl A, Krex D, Mohr P, Mauch C, Rass K, Bostroem A, et al. Number of metastases, serum lactate dehydrogenase level, and type of treatment are prognostic factors in patients with brain metastases of malignant melanoma. Cancer. 2011;117(8):1697-1703.

15. Fang C, Zhu T, Zhang P, Xia L, Sun C. Risk factors of neurosurgical site infection after craniotomy: A systematic review and meta-analysis. Am J Infect Control. 2017;45(11):e123-e134.

16. Kutteruf R, Yang JT, Hecker JG, Kinney GA, Furman MA, Sharma D. Incidence and risk factors for intraoperative seizures during elective craniotomy. J Neurosurg Anesthesiol. 2019;31(2):234-240.

17. Charlson ME, Pompei P, Ales KL, MacKenzie CR. A new method of classifying prognostic comorbidity in longitudinal studies: development and validation. J Chronic Dis. 1987;40(5):373-383.

18. Deyo RA, Cherkin DC, Ciol MA. Adapting a clinical comorbidity index for use with ICD-9-CM administrative databases. J Clin Epidemiol. 1992;45(6):613-619.

19. Liu H, Xu YB, Guo CC, Li MX, Ji JL, Dong RR, Zhang LL, et al. Predictive value of a nomogram for melanomas with brain metastases at initial diagnosis. Cancer Med.
2019;8(18):7577-7585.

20. Gardner LJ, Ward M, Andtbacka RHI, Boucher KM, Bowen GM, Bowles TL, Cohen AL, et al. Risk factors for development of melanoma brain metastasis and disease progression: a single-center retrospective analysis. Melanoma Res. 2017;27(5):477-484.

21. Li W, Xiao Y, Xu X, Zhang Y. A novel nomogram and risk classification system predicting the cancer-specific mortality of patients with initially diagnosed metastatic cutaneous melanoma. Ann Surg Oncol. 2021;28(7):34903500.

22. Yuan TA, Lu Y, Edwards K, Jakowatz J, Meyskens FL, Liu-Smith F. Race-, age-, and anatomic site-specific gender differences in cutaneous melanoma suggest differential mechanisms of early- and late-onset melanoma. Int J Environ Res Public Health. 2019;16(6):908.

23. Bellenghi M, Puglisi R, Pontecorvi G, De Feo A, Care A, Mattia G. Sex and gender disparities in melanoma. Cancers (Basel). 2020;12(7):1819.

24. Quan H, Li B, Couris CM, Fushimi K, Graham P, Hider $\mathrm{P}$, Januel JM, et al. Updating and validating the Charlson comorbidity index and score for risk adjustment in hospital discharge abstracts using data from 6 countries. Am J Epidemiol. 2011;173(6):676-682.

25. Lasithiotakis K, Zoras O. Metastasectomy in cutaneous melanoma. Eur J Surg Oncol. 2017;43(3):572-580.

26. Larkin J, Chiarion-Sileni V, Gonzalez R, Grob JJ, Cowey CL, Lao CD, Schadendorf D, et al. Combined nivolumab and ipilimumab or monotherapy in untreated melanoma. N Engl J Med. 2015;373(1):23-34.

27. Robert C, Grob JJ, Stroyakovskiy D, Karaszewska B, Hauschild A, Levchenko E, Chiarion Sileni V, et al. Fiveyear outcomes with dabrafenib plus trametinib in metastatic melanoma. N Engl J Med. 2019;381(7):626-636.

28. Margolin K, Atkins B, Thompson A, Ernstoff S, Weber J, Flaherty L, Clark I, et al. Temozolomide and whole brain irradiation in melanoma metastatic to the brain: a phase II trial of the Cytokine Working Group. J Cancer Res Clin Oncol. 2002;128(4):214-218.

29. Wollina U, Brzezinski P. The value of metastasectomy in stage IV cutaneous melanoma. Wien Med Wochenschr. 2019;169(13-14):331-338. 